Available online on 15.06.2020 at http://ajprd.com
(C) 2013-20, publisher and licensee AJPRD, This is an Open Access article which permits unrestricted non-
commercial use, provided the original work is properly cited

Open $\mathcal{O}_{\text {Access }}$

Review Article

\title{
Comparison Study of Various Nano Technology Formulation of Curcumin in Treatment of Alzheimer's Disease
}

\section{Mahaveer Singh 1 , Kushali $\mathbf{D}^{2}$, Vinay $\mathrm{C}^{\mathbf{1}}{ }^{1}$}

${ }^{1}$ Department of Pharmaceutics, D. R. Karigowda College of Pharmacy, Hassan, Karnataka -573 201.

2Department of Pharmaceutics, Saradavilas College of Pharmacy, Mysuru, Karnataka -570 004

\begin{abstract}
A B S T R A C T
Alzheimer's disease (AD) is a progressive neurodegenerative disorder associated with cognitive deterioration affecting day to day living and behavioural activities. It is commonest cause of senile and pre-senile dementia. In Alzheimer's disease, a peptide referred as amyloid beta aggregates (oligomers), and accumulates in the brain to form deposits called as amyloid plaques. According to the world health organization (WHO), $5 \%$ of men and $6 \%$ of women of above 60 years are victims with Alzheimer's type dementia globally. In India prevalence of dementia is $33.6 \%$, of which AD contributes approximately $54 \%$ and vascular dementia constitutes approximately 39\%. Patients with the prolonged use of some Non-steroidal anti-inflammatory (NSAID) drugs such as ibuprofen have lower risk of developing the symptoms of AD; however the chronic use of NSAID can produce a toxic effect on the kidney, liver and GI tract. Recent studies have demonstrated that a curcumin delivery system based on nanoscience and nanotechnology increases the therapeutic potential of this compound. Specifically, several nano-sized carriers such as phospholipid vesicles (liposomes), micelles, solid lipid nanoparticles, polymeric nanoparticles, emulsions, proteins and other molecular complexes have been developed for the efficient delivery of curcumin. Nano particles have demonstrated the enhanced Bioavailability of curcumin including circulation time in the blood. Various nano technology drug delivery system of curcumin showed increased Bio accessibility during treatment of Alzheimer's disease.
\end{abstract}

Key words: Alzheimer's disease, Dementia, Curcumin, Nano Emulsion, Nano Gel, Nano Suspension, Liposomes.

\footnotetext{
A R T I C L E I N F 0: Received 08 April 2020; Review Completed 27 May 2020; Accepted 05 June 2020; Available online 15 June. 2020

Cite this article as:

Singh M, Kushali D, Vinay C H, ${ }^{1}$ Department of Pharmaceutics, D. R. Karigowda College of Pharmacy, Hassan, Karnataka -573

201., Asian Journal of Pharmaceutical Research and Development. 2020; 8(3):99-103.

DOI: http://dx.doi.org/10.22270/ajprd.v8i3.743

*Address for Correspondence:

Mahaveer singh, Assistant Professor, Department of Pharmaceutics, D.R. Karigowda College of Pharmacy,Kuvempu Nagar, Hassan, Karnataka, India.
}

\section{INTRODUCTION:}

$\sim$ urcumin1, 7bis (4hydroxy3methoxyphenyl)1,6 heptadiene-3,5-dione)is found in the lipophilic extract of the rhizomes of turmeric (Curcuma longa). Numerous reports suggest that this natural compound possesses therapeutic benefit such as antitumour, anti-inflammatory and anti-oxidant activities. The bioavailability of conventional oral curcumin is low. Therefore the development of an efficient drug delivery system for curcumin is of considerable interest ${ }^{1}$.

Alzheimer's disease (AD) is the commonest progressive neurodegenerative disorder of elderly population, characterized by memory dysfunction, spatial and temporal disorientation and impairment of judgment clinically. The pathogenesis of AD is characterized by loss of neurons and synapses, resulting in gross atrophy across multiple brain regions. The disease is presently hard to reliably diagnose, particularly in its early stages. While an accurate diagnosis can only be obtained post-mortem(via examination of brain tissue in an autopsy), current clinical methods involving brain imaging and neuropsychological testing are only about $85 \%$ accurate, that to only at later stages ${ }^{2}$.

Clinical development of drugs for the symptomatic and disease-modifying treatment of $\mathrm{AD}$ has generated in both promise and disappointment ${ }^{3}$. In $\mathrm{AD}$, Biochemical mediators in the inflammation cascade contribute both to neurodegeneration and production as well as accumulation 
of the $\beta$-amyloid peptide, including interleukin (IL)- $1 \beta$, phospho-c-Jun NH2-terminal kinase (pJNK), reactive oxygen species, inducible nitric-oxide synthase (iNOS) and lipid peroxidation products (e.g., 8 -iso-PGF $2 \alpha)^{4}$. The neuropathlogical process in AD consists of neuronal loss and atrophy neurotic plaques are relatively insoluble dense cores of 5-10 nm thick amyloid fibrils with a pallor staining "halo" surrounded by dystrophic neuritis, reactive astrocytes and activated microglia. There is an increased presence of monocytes/macrophages in the cerebral vessel wall and reactive or activated microglia cells in the adjacent parenchyma. The main protein component of amyloid in $\mathrm{AD}$ is the 39-42 amino acid (beta) amyloid peptides (Abeta) ${ }^{5}$.

Curcumin has broad-spectrum of therapeutic activities. The low bioavailability of curcumin and poor water solubility limits its medicinal value ${ }^{6} . " N a n o t e c h n o l o g y-e n h a n c e d$ curcumin: symbiosis of ancient wisdom of the east with modern medical science". In the context of AD pathology, in-vitro as well as animal model studies demonstrate that curcumin can have beneficial effects through multiple pathways, such as its strong anti-oxidant activity ${ }^{7-8}$. Interaction with metal ions and preventing metal-induced aggregation of beta-amyloid 9-10 and destabilizing preformed beta-amyloid fibrils as well as inhibiting $A \beta$ aggregate formation ${ }^{11-13}$. Thus, administration of curcumin is being seen as a potent therapeutic (and preventive) strategy against $\mathrm{AD}^{14}$. Nano robot has also been developed for effective cures of disorders which are not amenable to treat. Principles from photodynamic therapy, fullerene chemistry, nano structuring, X-rays, computers, pharmacokinetics and robotics are applied in the design of nanorobot for treatment of Alzheimer's disease ${ }^{15}$.
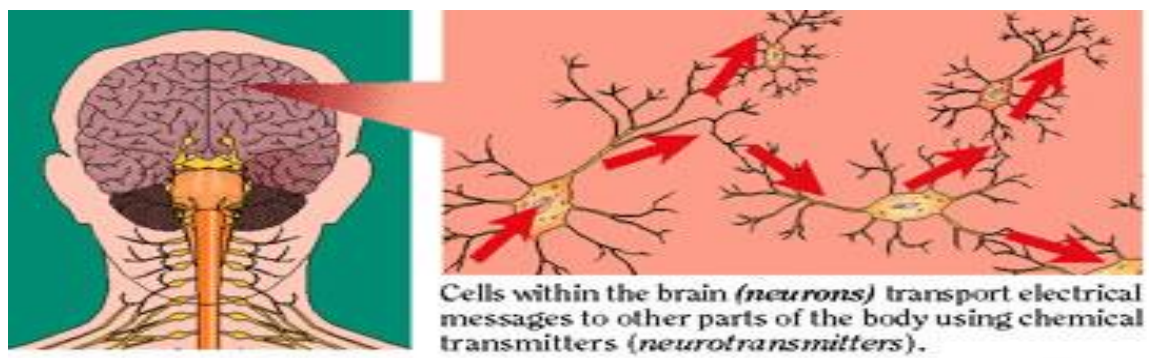

Cells within the brain (neuroms) transport electrical messages to other parts of the body using chemical transmitters (nezsotransmitters).
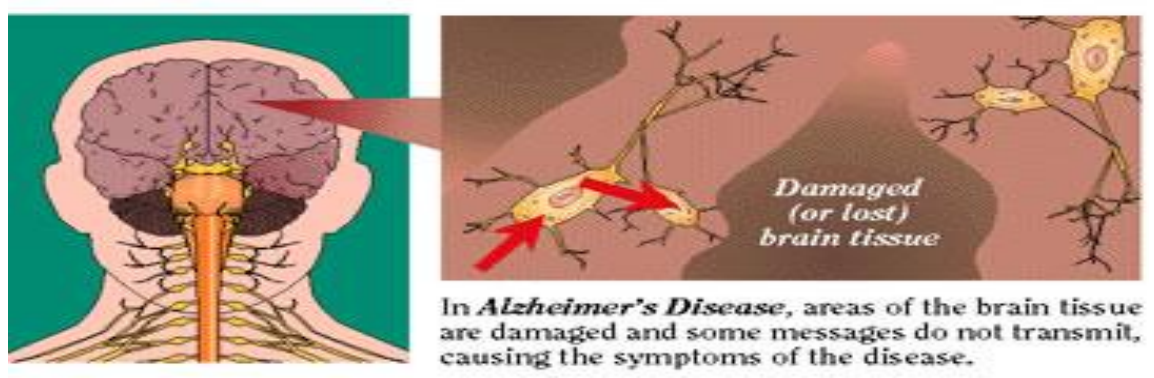

In ALzheimer's Disease, areas of the brain tissue are damagjed and some messages do not transmit, causing the symptoms of the disease.

Figure 1:Conditon of brain in normal and alzheimers patient

\section{Curcumin and Alzheimer's disease:}

Globally about 1000 published animal and human studies, both in-vivo and in-vitro on the effects of curcumin on various diseases have been studied inclusive of epidemiological, basic and clinical research on $\mathrm{AD}^{16}$.

\section{Mechanism of action of Curcumin on Alzheimer's disease:}

$\mathrm{AD}$ deteriorates the nerve cells which might involve $\beta$ ammation, oxidative damage and mostly observed the formation of beta-amyloid plaques, metal toxicity.
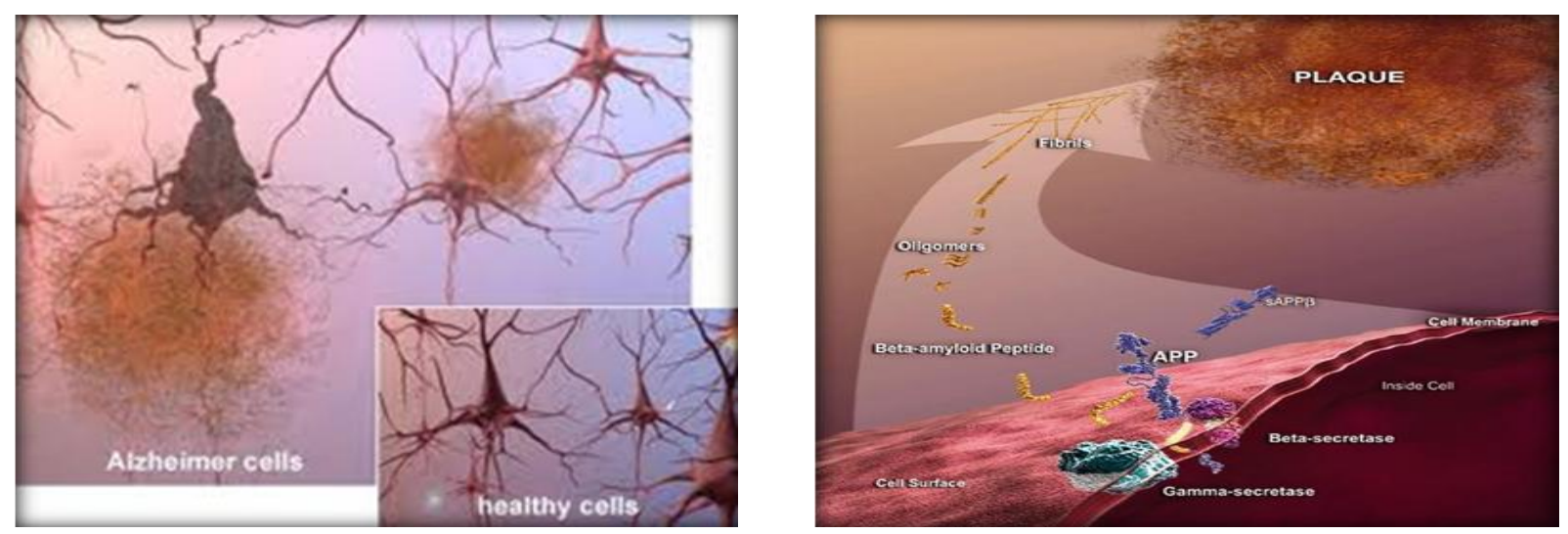

Figure 2: Formation of plaque in brain 


\section{Effects of Curcumin on macrophages:}

A study carried at UCLA observed that curcumin might help the macrophages to scavenge the amyloid plaques which are observed in Alzheimer's disease. Macrophages play key role in the immune system. They help the body to fight against foreign proteins to effectively eliminate them.

Curcumin was treated with macrophages in blood taken from nine volunteer's six AD patients and three healthy controls. Beta amyloid was then injected. The AD patients, whose macrophages were treated with curcumin, when compared with patients whose macrophages were not treated with curcumin, showed an improved uptake and ingestion of the plaques. Thus, curcumin may support the immune system to clear the amyloid protein ${ }^{17}$.

The most marked characteristic feature in $\mathrm{AD}$ is the presence of beta-amyloid plaques, which are basically an accumulation of small fibers called beta amyloid fibrils. Because the deposition of beta amyloid protein is a consistent pathological trade mark of brains affected by $\mathrm{AD}$, the inhibition of A-beta generation, prevention of Abeta fibril formation, destabilization of preformed A-beta would be a fascinating therapeutic approach for the treatment of $\mathrm{AD}$. The levels of beta-amyloid in $\mathrm{AD}$ mice that were given low doses of curcumin were lowered approximately by $40 \%$ compared to those that were not treated with curcumin. Additionaly, low doses of curcumin also reduced plaque formation by $43 \%$. Surprisingly low doses of curcumin administered over longer period were more effective than high doses in amelioration of neurodegenerative process of AD. At higher concentration, curcumin binds to amyloid beta and blocks its selfassembly ${ }^{18}$. The important chemical features in amyloid beta are the presence of two aromatic end groups and any changes in these groups has profound effect on its activity $^{20}$. A new biosynthesis of nanoparticles is an interesting method that has attracted significant focus due to their potential use in many applications, such as catalysis, drug delivery biosensor, Anti-microbial and therapeutics. Biological methods of nanoparticles synthesis employing microorganism, enzyme, and plant or plant extract have been proposed as feasible environment friendly alternatives to chemical and physical methods ${ }^{19}$. About 40 different nanostructuring operations were reviewed recently. Latest developments in nanomedicine were reviewed.

\section{Development of Nano technology:}

Submarine Nano robots are being developed for use in spinal surgery, cancer therapy, etc. Nanoparticles have been developed for use in drug delivery systems and for cure in eye disorders and in early diagnosis. Investigation in nanomedicine is under development of diagnostics for rapid monitoring, targeted cancer therapies, localized drug delivery, and improved cell material interactions, scaffolds for tissue engineering and gene delivery systems.

\section{Nano carrier for Curcumin delivery:}

Unsatisfactory pharmacokinetics of oral administration is often observed for poorly water-soluble drug candidates. In this case, drug delivery systems which permit systemic injection of insoluble drugs are particularly useful. Fortunately, the hydrophobicity of curcumin provides an effective strategy to stably fix curcumin in a hydrophobic domain of various types of nanoparticles. Drug delivery carriers are often classified according to their size (i.e., micro- or nano-) because this is an important factor in characterizing the system ${ }^{20}$. Nanotechnology-based drug delivery systems for curcumin including liposomes, micelles, Nano gels, Nano Suspension, have been attempted to enhance the oral bioavailability, biological activity or tissue-targeting ability of curcumin ${ }^{21}$.

\section{The Nano Emulsion based delivery of Curcumin:}

Nano Emulsion have attracted great attention in research, dosage form design and pharmacotherapy. This is as a result of a number of attributes peculiar to nano suspension such as optical clarity, ease of preparation, thermodynamic stability and increased surface area ${ }^{22}$.

Nanoemulsions can be prepared by high- and low-energy methods. Both high-energy and low- energy methods can produce stable Nanoemulsions. High-pressure homogenizer or ultra- sound generator can be used for the preparation of nanoemulsion by high-energy emulsification method. Selfemulsification and phase inversion methods-phase inversion temperature and phase inversion composition are low-energy methods for the preparation of nanoemulsions. Low-energy emulsification methods depend on the phase behaviour and properties of the constituents, and they utilize the stored energy of the system to form nano droplets. The emulsification can be brought about by changing the parameters such as temperature and composition, which would affect the hydrophilic lipophilic balance of the system.

Nanoemulsions offer several advantages for the delivery of drugs and are thus receiving increasing attention as drug carriers for improving the delivery of active pharmaceutical ingredient curcumin in treatment of Alzheimer's disease ${ }^{23}$. Self-Nano emulsification is only achieved with some oils, surfactants, and co-surfactants at a certain ratio. Selection of an appropriate oily phase is very important, as it influences the selection of other ingredients in oil/water nanoemulsions, generally, surfactant alone cannot lower the oil interfacial tension sufficiently to yield a nanoemulsion; this necessitates the addition co-surfactant to lower the surface tension close to zero ${ }^{24}$.

\section{The Nano Suspension based delivery of Curcumin:}

The curcumin nanosuspension on a lab scale is typically produced by pre-milling (with SDS $0.2 \%$ ) followed by high pressure homogenization in pure water using a con- tinuous Micron LAB 40 at room temperature, applying 20 homogenization cycles at 1500 bar. Curcumin obtained through nanosuspension over normal/commercial curcumin in being more bio-efficient, suggesting novel delivery strategies for curcumin in therapeutic applications ${ }^{20}$.the highly stabilized dried nano suspension of curcumin (mean particle size, $<80 \mathrm{~nm}$ ) formulation with remarkable reproducibility and ease of storage. Pharma-cokinetic studies showed that the nanoparticle formulation 
significantly improved curcumin bioavailability, with a much greater plasma concentration and six fold higher AUC and MRT in brain. This novel nano suspension curcumin may have great potential for AD therapy ${ }^{25}$. This study offers significant promises and is worthy of further exploration in attempts to enhance the bioavailability, medicinal value, and application of this interesting molecule from mother nature ${ }^{20}$.

\section{The Nano Gels based delivery of Curcumin:}

Nanogel served as an effective "Nano carrier" for the formulation of lipophilic curcumin by increasing its water solubility, improving its stability, and controlling its release profile $^{26}$.

The work of Ikeda et al. is an example for the $A \beta$ antiassembly strategy. They designed an amphipathic Nano gels that incorporates proteins and controls their folding and aggregation, similar to natural chaperones (proteins assisting the non-covalent folding and/or unfolding). In the case of $A \beta$, these Nano gels would inhibit the amyloid genesis process effectively through this mechanism ${ }^{27}$. But Incorporation of cur-cumin into nanogel did not compromise its cytotoxicity.

\section{The Liposome based delivery of Curcumin:}

Liposomes are the most studied nanoparticles for drug delivery. In general, physically stable liposomes are formulated with several kinds of lipid, where the first step is the preparation of the mixed lipids the average diameter of liposomes for typical drug delivery applications is 50$300 \mathrm{~nm}$. It has been confirmed that the lipophilic curcumin is located within the membranes of the liposomes but is absent from the inner aqueous phase the curcumin may be located on the surface of membrane at the hydrophilic/hydrophobic interface, or within the hydrophobic domain. Experimental evidence suggests curcumin is located in the hydrophobic domain of the lipid bilayer membrane ${ }^{1}$. Liposomes are of a great importance as nano-carriers due to their relatively large carrying capacity. They have long been used as drug delivery system to the brain, because the particles can entrap the compounds and prevent the rapid elimination or degradation as well as promote the penetration through the BBB which in turn decreases the effective dose among all the applications of

\section{REFERENCES:}

1. Sou K. Curcumin towards nanomedicine. Rec Pat Nanomed. 2012; 2(2):133-45.

2. Knopman DS, DeKosky ST, Cummings JL, Chui H, Corey-Bloom J, Relkin N, Small GW, Miller B, Stevens JC. Neurology. 2001; 56(9):1131-2.

3. Sahni JK, Doggui S, Ali J, Baboota S. Alzheimer's disease. J Controlled Rele; 2011; 152(2):208-31.

4. Aynun N, Begum, Mychica R. Jones, Giselle P, Limet. Curcumin structure-function, bioavailability, and efficacy in models of neuroinflammation and alzheimer's disease. J Pharmacol Exp Ther. 2008; 326(1):196-208.

5. Mishra S, Kalpana, Palanivelu. The effect of curcumin (turmeric) on Alzheimer's disease: An overview. Ann Indian Acad Neurol. 2008; 11(1):13-9. liposomal technology, the development of a suitable liposomal carrier to encapsulate neuroactive compounds is very promising. These liposomes are stable enough to be carried to the brain across the BBB, with the appropriate surface characteristics for an effective targeting and for an active membrane transport. Improvements and adjustments to the liposomal formulation are constantly being explored through the addition of different lipids and targeting molecules. For example, in liposomes lacking cholesterol, high-density lipoprotein can cause disintegration of the liposome, or in liposomes which do contain too much cholesterol, high-density lipoprotein canal so cause leakage of contents. The development of novel therapeutic strategies for neurodegenerative and neurological diseases represents one of the biggest unmet medical needs today ${ }^{28}$.

\section{CONCLUSION:}

1. Nano emulsification is only achieved with some oils, surfactants, and co-surfactants at a certain ratio. Selection of an appropriate oily phase is very important, as it influences the selection of other ingredients in oil/water nanoemulsions, generally, surfactant alone cannot lower the oil interfacial tension sufficiently to yield a nanoemulsion.

2. Nano Suspension offers significant promises and is worthy of further exploration in attempts to enhance the bioavailability, and various medicinal value

3. Nano gels would inhibit the amyloid genesis process effectively through this mechanism. But Incorporation of curcumin into nanogel did not compromise its cytotoxicity.

4. Improvements and adjustments to the liposomal formulation are constantly being explored through the addition of different lipids and targeting molecules. But in liposomes lacking cholesterol, high-density lipoprotein can cause disintegration of the liposome, or in liposomes which do contain too much cholesterol, high-density lipoprotein canal so cause leakage of contents.

5. Based on the above details this novel nano-Suspension delivery of curcumin may have great potential for $\mathrm{AD}$ therapy and can be a reference for future studies on formulation of drugs as nano-suspension.

6. Lee W, Loo C, Bebawy M, Luk F, Mason SR, Rohanizadeh R. Curcumin and its derivatives: their application in neuropharmacology and neuroscience in the 21 st century. Current Neuropharmaco. 2013; 11:338-78.

7. Mahajan YR. Nanotechnology-enhanced curcumin: symbiosis of ancient wisdom of east with modern medical science. Nanotech Insights. 2011; 2:17-27.

8. Ringman JM. A potential role of the curry spice curcumin in alzheimer's disease. Curr Alzheimer Res. 2005; 2(2):131-36.

9. Daniel S. Through metal binding, curcumin protects against leadand cadmium-induced lipid peroxidation in rat brain homogenates and against lead- induced tissue damage in rat brain. $\mathrm{J}$ Inorg Biochem. 2004; 98(2):266-75.

10. Baum L, Ng A. Curcumin interaction with copper and iron suggests one possible mechanism of action in alzheimer's disease animal models. J Alzheimer's Dis. 2004; 6(4):367-77. 
11. Yang F. Curcumin inhibits formation of amyloidbeta oligomers and fibrils, binds plaques, and reduces amyloid in-vivo. J Biol Chem. 2005; 280(7):5892-901.

12. Garcia-Alloza M. Curcumin labels amyloid pathology In-Vivo, disrupts existing plaques and partially restores distorted neurites in an alzheimer mouse model. J Neurochem. 2007; 102(4):1095-104.

13. Ono K. Curcumin has potent anti-amyloidogenic effects for alzheimer's beta fibrils in-vitro. J Neurosci Res. 2004; 75(6):742-50.

14. Mishra S, Palanivelu K. The Effect of curcumin (turmeric) on alzheimer's disease: an overview. Ann Indian Acad Neurol. 2008; 11(1):13-9.

15. Luo Z, Rohan V, Tikekar, Nitin N. Chemistry approach for imaging intracellular and intratissue distribution of curcumin and its nanoscale carrier. Bioconju Chemi. 2014; 25(1):32-42.

16. Pandav R, Belle SH, Dekosky ST. Apolipoprotein E polymorphism and Alzheimer s disease: The Indo-US cross-national dementia study. Arch Neural 2000; 57:824-30.

17. Ng TP, Chiam PC, Lee T, Chua HC, Lim L, Kua EH. Curry consumption and cognitive function in the elderly. Am J Epidemiol. 2006; 164(9):898-906.

18. Yang F, Lim GP, Begum AN, Ubeda OJ, Simmons MR, Ambegaokar SS. Curcumin inhibits formation of amyloid beta oligomers and fibrils, binds plaques, and reduces amyloid in-vivo.J Biol Chem. 2005; 280(7):5892-901.

19. Elumalai S, Devika R. Biosynthesis of silver nanoparticles using curcuma longa and their antibacterial activity. Int J Pharm Res Sci. 2014; 2(1):98-103.
20. Ravichandran R, Pharmacokinetic study of nanoparticulate curcumin: oral formulation for enhanced bioavailability. J Biomat Nanobiotec. 2013; 4(3):291-99.

21. Sou K. Curcumin towards nano medicine. Recent Patent Nano Medi. 2012; 2(2):133-45.

22. Lovelyn C, Attama AA. Current state of nanoemulsions in drug delivery. J Biomat Nanobiotech. 2011; 2:626-39.

23. Chime SA, Kenechukwu FC, Attama AA, Nanoemulsions advances in formulation, characterization and applications in drug delivery. Intech.

24. Rachmawati H, Budiputra DK, Suhandono S, Anggadiredja K. Curcumin nanoemulsion for transdermal application:formulation and evaluation, research and development on nanotechnology in Indonesia. 2014;1(1):5-8.

25. Cheng KK, Yeung CF, Ho SW, Chow SF, Chow AH, Baum L. Highly stabilized curcumin nanoparticles tested in an in vitro bloodbrain barrier model and in alzheimer's disease Tg2576 mice. AAPS J. $2013 ; 15(2) .324-36$.

26. Gonçalves C, Pereira P, Schellenberg P, Coutinho PJ, Gama FM. Self-assembled dextrin nanogel as curcumin delivery system. J Biomater Nanobiotech. 2012; 3(2):178-84.

27. Nazem A, Mansoori GA. Nanotechnology for Alzheimer's disease detection and treatment. Insciences J. 2011; 1(4):169-93.

28. Spuch C, Navarro C. Liposomes for targeted delivery of active agents against neuro degenerative diseases (alzheimer's disease and parkinson'sdisease). J Drug Delivery. 2011. 